past, little religious value, and tends to a contentment with less than the full gamut of religious experience. No one of the three forms of God-experience can be dispensed with in a rich and fruitful spiritual life; and it is no wonder that the orthodox have generally felt a merely negative Unitarianism to have an impoverishing tendency. However crude the creedal affirmations of Trinitarianism may be, the fulness of the Christian life has by it been fostered and preserved. So, however loath we may be to seem to accept the description of a quasi-human Being who is somehow Three Persons and yet One, if we take the doctrine (as we must take all religious doctrines) in its inner and spiritual sense, which is its empirical foundation-sense, we shall see it as a more or less blind expression of a great truth-that Christians can attain to the vision of God in three ways, through contemplation of the outer world, through faith in their Master Christ, and through obedience to the Holy Spirit in their hearts.

Durant Drake.

Vassar College.

\title{
GENERAL NOTES ON THE CONSTRUCTION OF MAGIC SQUARES AND CUBES WITH PRIME NUMBERS.
}

The series of numbers generally used in the construction of magic squares are in arithmetical progression. The progression of the prime number series is very irregular, and therefore cannot be used as freely as an arithmetical series. This naturally leads to the investigation of the possible irregularities in groups or series of numbers which may be formed into magic squares. It is also necessary to find means of discovering these groups of numbers in the prime number series.

It is the writer's aim to describe here simple rules for constructing prime number squares, methods of finding the numbers to be used, and to point the way to the solution of a few of the problems not yet mastered.

THE SQUARE OF THE 3d ORDER.

There is only one possible construction of this square and there is only one rule governing the series, and that is, when the series is written in tabular form, as in Fig. 1, the differences between all 
vertically adjacent cells must be equal, and the differences between all horizontally adjacent cells must be equal, but the vertical and horizontal differences must be unequal to avoid duplicate numbers. These differences are indicated by numbers at the sides of the lattice and it will be by these that we will identify the nature of the series

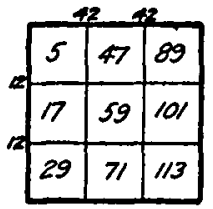

Fig. 1.

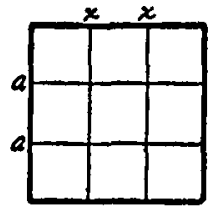

Fig. 2.

\begin{tabular}{|c|c|c|}
\hline 71 & 5 & 101 \\
\hline 89 & 59 & 29 \\
\hline 17 & 113 & 47 \\
\hline
\end{tabular}

Fig. 3.

used in the following magic squares. We will represent these differences by letters, using the letters of the fore part of the alphabet for one set of differences and those of the other end of the alphabet for the other set, as is shown in Fig. 2, like letters indicating the necessity of like differences.

Fig. 2 is arranged into the magic by using the middle column and middle line as diagonals, the position of the remaining numbers then being easily found. The resulting magic is shown in Fig. 3.

THE SQUARE OF THE 4th ORDER.

Any series or set of 16 numbers, when written in the tabular form previously mentioned, wihch gives the differences $a, b, c$ and $x, y, z$, may be formed into a magic square by the Jaina method as follows. Fig. 4 shows a table of prime numbers with irregular differences. Four sets of the upper line of numbers of this table are arranged

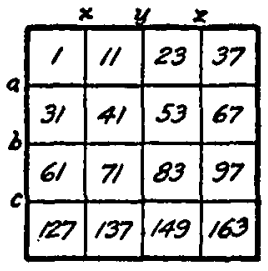

Fig. 4.

\begin{tabular}{|c|c|c|c|}
\hline 1 & 11 & 23 & 37 \\
\hline 23 & 37 & 1 & 11 \\
\hline 37 & 23 & 11 & 1 \\
\hline 11 & 1 & 37 & 23 \\
\hline
\end{tabular}

Fig. 5.

\begin{tabular}{|c|c|c|c|}
\hline$\prime$ & 71 & 149 & 67 \\
\hline 53 & 163 & 61 & 11 \\
\hline 97 & 23 & 41 & 127 \\
\hline 137 & 31 & 37 & 83 \\
\hline
\end{tabular}

Fig. 6.

in a subsidiary square, as shown in Fig. 5, so that each line, column, and the two diagonals contain each of the four different numbers. Subtracting the initial number of the table (in this case 1) from each of the numbers in the left-hand column of the table, will give the numbers, $0,30,60,126$, which are to be arranged in a second subsidiary square with the same arrangement as in Fig. 5, only that 
the pattern is turned 90 degrees. The two subsidiary squares are then added together, cell to cell, to produce the magic square. A resulting square is shown in Fig. 6.

In selecting numbers from the tables for the subsidiary squares, the column and line containing the lowest numbers should be chosen, but it makes no difference which set the initial number is subtracted from.

A balanced series of numbers whose tabular differences are

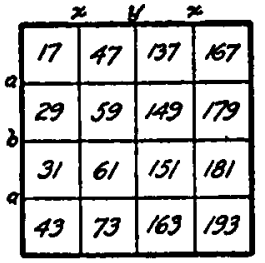

Fig. 7.

\begin{tabular}{|c|c|c|c|}
\hline 193 & 47 & 197 & 49 \\
\hline 29 & 151 & 61 & 179 \\
\hline 31 & 149 & 59 & 181 \\
\hline 167 & 73 & 163 & 17 \\
\hline
\end{tabular}

Fig. 8.

\begin{tabular}{|c|c|c|c|}
\hline 17 & 73 & 149 & 181 \\
\hline 179 & 151 & 47 & 43 \\
\hline 61 & 29 & 193 & 197 \\
\hline 163 & 167 & 31 & 59 \\
\hline
\end{tabular}

Fig. 9.

$a, b, a$, and $x, y, x$, may be arranged into an associated square, or a pandiagonal square. Such a series is shown in Fig. 7. By revolving the two diagonals of Fig. 7, 180 degrees, it will produce the associated magic square shown in Fig. 8. To produce a pandiagonal square, we select, as before mentioned, two subsidiary sets of numbers from which are formed two subsidiary squares of the pattern shown in Fig. 5. The numbers in the upper line should be so arranged that the sum of the left-hand pair equals the sum of the right-hand pair. One of these subsidiary squares is revolved 90 degrees and added to the other to produce the magic. A pandiagonal square resulting from such a construction is shown in Fig. 9.

Another form of subsidiary square which may be used to produce a pandiagonal square from a balanced series is shown in Fig. 10, which is exemplified with arbitrary numbers. The numbers

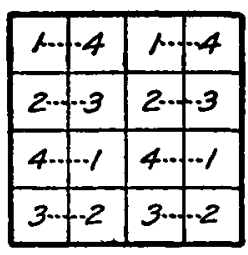

Fig. 10.

\begin{tabular}{|c|c|c|c|}
\hline 167 & 163 & 77 & 79 \\
\hline 29 & 61 & 179 & 151 \\
\hline 193 & 137 & 43 & 47 \\
\hline 31 & 59 & 181 & 149 \\
\hline
\end{tabular}

Fig. 11.

must be so arranged that the pairs indicated by dotted lines will have like summations. One subsidiary square is revolved 90 degrees from the other and the two added together to produce the final 
square. Fig. 11 is a pandiagonal square produced from the series in Fig. 7 , by the method last described.

THE SQUARE OF THE 5th ORDER.

A series of 25 numbers whose tabular differences are $a, b, c, d$ and $w, x, y, z$ is shown in Fig. 12. Such a series may be formed

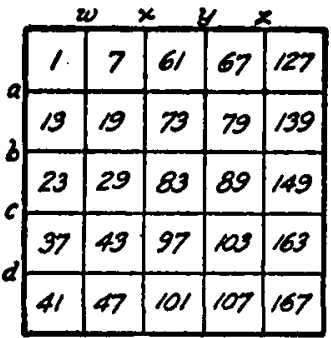

Fig. 12.

\begin{tabular}{|l|l|l|l|l|}
\hline 1 & 2 & 3 & 4 & 5 \\
\hline 4 & 5 & 1 & 2 & 3 \\
\hline 2 & 3 & 4 & 5 & 1 \\
\hline 5 & 1 & 2 & 3 & 4 \\
\hline 3 & 4 & 5 & 1 & 2 \\
\hline
\end{tabular}

Fig. 13.

\begin{tabular}{|c|c|c|c|c|}
\hline 1 & 29 & 101 & 79 & 103 \\
\hline 73 & 103 & 127 & 23 & 47 \\
\hline 149 & 41 & 19 & 97 & 67 \\
\hline 43 & 61 & 89 & 167 & 13 \\
\hline 107 & 199 & 37 & 7 & 85 \\
\hline
\end{tabular}

Fig. 14.

into a pandiagonal square as follows. Two sets of subsidiary numbers are selected and arranged in two subsidiary squares according to the pattern shown in Fig. 13 ; the pattern of one subsidiary square should, however, be in a reversed or reflected order from the other. The two squares are then united to form the final square. Fig. 14 shows one example resulting from the series in Fig. 12.

To produce an associated pandiagonal square of the 5 th order, it requires a series whose tabular differences are $a, b, b, a$ and $x, y, y, x$. The writer, at present, knows of only one series which suits the above requirements. Its initial number is 41 and the tabular differences are $60,390,390,60$ and $72,138,138,72$ respec-

\begin{tabular}{|c|c|c|c|c|}
\hline 1013 & 251 & 449 & 911 & 881 \\
\hline 839 & 1301 & 941 & 113 & 311 \\
\hline 41 & 173 & 701 & 1229 & 1961 \\
\hline 1091 & 1289 & 461 & 101 & 563 \\
\hline 521 & 491 & 953 & 1151 & 389 \\
\hline
\end{tabular}

Fig. 15.

tively. The subsidiary squares are arranged in associated formation and according to the pattern in Fig. 13. The solution of this difficult problem was accomplished by Mr. Chas. D. Shuldham, and his resulting magic is shown in Fig. 15.

Mr. Shuldham has succeeded by other methods in constructing 
associated squares of the various orders including the 12th. ${ }^{1}$ These are not pandiagonal however.

THE SQUARE OF THE 6th ORDER.

A balanced series whose tabular differences are $a, b, c, b, a$ and

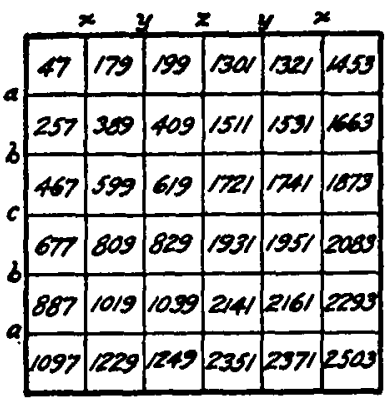

Fig. 16.

\begin{tabular}{|l|l|l|l|l|l|}
\hline 1 & 6 & 6 & 6 & 1 & 1 \\
\hline 5 & 2 & 5 & 2 & 2 & 5 \\
\hline 3 & 4 & 3 & 3 & 4 & 4 \\
\hline 4 & 3 & 4 & 4 & 3 & 3 \\
\hline 2 & 5 & 2 & 5 & 5 & 2 \\
\hline 6 & 1 & 1 & 1 & 6 & 6 \\
\hline
\end{tabular}

Fig. 17.

$x, y, z, y, x$ may, by a common method, be formed into a magic square as follows. Fig. 16 shows a series with the above differences. It will be noted that the values for $a, b$ and $c$ are each 210 , though the method of construction does not necessitate these like differences. Subsidiary sets of numbers are selected as previously explained and formed into subsidiary squares according to the pattern shown in Fig. 17. One of the squares thus formed is revolved 90 degrees and added to the other, cell to cell, to form the final square. One

\begin{tabular}{|c|c|c|c|c|c|}
\hline 4 & 2203 & 2003 & 1878 & 257 & 1097 \\
\hline$|x z|$ & o & 1851 & 595 & 1010 & 2371 \\
\hline 20 & |SוI & 619 & 829 & $2 \mu \mid$ & 1001 \\
\hline 2381 & 980 & $m 2$ & 1981 & 100 & 199 \\
\hline 20 & 1531 & 809 & 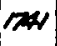 & 261 & 179 \\
\hline 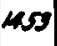 & 1007 & 467 & 677 & $16 \omega$ & 50 \\
\hline
\end{tabular}

Fig. 18.

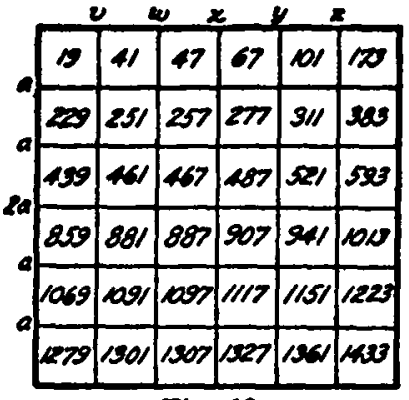

Fig. 19.

example is shown in Fig. 18, which is magic only in its lines, columns and two diagonals.

A series of prime numbers to suit the above differences is very difficult to find, but the following form of series is easily found, and it is only recently that the writer has succeeded in discovering a

1 See "Associated Prime Number Magic Squares," The Monist, July, 1914, Vol. XXIV, No. 3. 
method of arranging the numbers of such a series in magic square formation.

Fig. 19 shows a series whose tabular differences are $a, a, 2 a, a, a$ and $v, w, x, y, z$. The subsidiary numbers with differences corresponding to the $a$ values are arranged as shown in Fig. 20, each

\begin{tabular}{|c|c|c|c|c|c|}
\hline 1260 & 1260 & 1260 & 0 & 0 & 0 \\
\hline 210 & 210 & 210 & 1050 & 1050 & 1050 \\
\hline 420 & 420 & 420 & 840 & 840 & 840 \\
\hline 210 & 210 & 210 & 1050 & 1050 & 1050 \\
\hline 1260 & 1260 & 1260 & 0 & 0 & 0 \\
\hline 420 & 120 & 420 & 840 & 840 & 840 \\
\hline
\end{tabular}

Fig. 20.

\begin{tabular}{|c|c|c|c|c|c|}
\hline 19 & 41 & 47 & 67 & 101 & 179 \\
\hline 101 & 67 & 19 & 173 & 47 & 41 \\
\hline 41 & 47 & 173 & 19 & 67 & 101 \\
\hline 47 & 173 & 41 & 101 & 19 & 67 \\
\hline 173 & 101 & 67 & 47 & 41 & 19 \\
\hline 67 & 19 & 101 & 41 & 173 & 47 \\
\hline
\end{tabular}

Fig. 21.

line made up of three like pairs. The other set of subsidiary numbers is arranged as shown in Fig. 21, with a full set of the six different numbers in each line, column, the two central diagonals, and in the cells corresponding to the like numbers in Fig. 20. These two subsidiary squares combined, will produce the magic square shown in Fig. 22.

\begin{tabular}{|l|l|l|l|l|l|}
\hline 1279 & 1301 & 1307 & 67 & 101 & 173 \\
\hline 311 & 277 & 229 & 1223 & 1097 & 1091 \\
\hline 461 & 467 & 593 & 259 & 907 & 941 \\
\hline 257 & 989 & 251 & 1151 & 1069 & 1117 \\
\hline 1433 & 1361 & 1327 & 47 & 41 & 19 \\
\hline 487 & 499 & 521 & 881 & 1013 & 887 \\
\hline
\end{tabular}

Fig. 22.

To construct an associated or pandiagonal square of the 6th order would require a table of numbers with the differences $a, b$, $c, b, a$ and $x, y, z, y, x$ and each set of subsidiary numbers would have to be of values permitting thier arrangement in magic $2 \times 3$ rectangles. $^{2}$ It is doubtful if a series of this nature can be found, but, by methods previously published, the problem may be solved

2 To construct, see "Notes on the Construction of Magic Squares" by Messrs. Andrews and Frierson, The Monist, April 1912, Vol. XXII, No. 2. For an example, try any series with the tabular differences 2, 1, 4, 1, 2 and $9.11,18,11,9$, with the illustrations on p. 306 (of the above issue) as a guide. 
by first forming an associated square and transforming it into a pandiagonal square. ${ }^{8}$

THE SQUARE OF THE 7th ORDER.

This square, like the 5 th order square, may be formed into a pandiagonal magic by using a series having tabular differences of

\begin{tabular}{|c|c|c|c|c|c|c|c|}
\hline 17 & 41 & 17 & 347 & 613 & 859 & 1997 & 2693 \\
\hline 29 & 47 & 179 & 353 & 619 & 859 & 2003 & 2699 \\
\hline 227 & 251 & 383 & 557 & 823 & 1063 & 2207 & 2903 \\
\hline 233 & 257 & 389 & 563 & 829 & 1069 & 2213 & 2909 \\
\hline 443 & 467 & 599 & 773 & 1039 & 1279 & 2423 & 3119 \\
\hline 653 & 677 & 809 & 983 & 1249 & 1489 & 2633 & 3329 \\
\hline 857 & 881 & 1013 & 1187 & 1453 & 1693 & 2837 & 3533 \\
\hline 863 & 887 & 1019 & 1193 & 1459 & 1699 & 2843 & 3539 \\
\hline
\end{tabular}

Fig. 23.

$a, b, c, d, e, g$ and $t, v, w, x, y, z$. The subsidiary squares have like numbers running in knight paths, the pattern of one may be a reflection of the other, or they may have the same pattern, but 90 degrees apart.

THE SQUARE OF THE 8th ORDER.

This square may be formed with an irregular series having tabular differences of $a, b, c, d, e, g, h$ and $s, t, v, w, x, y, z$. One series is shown in Fig. 23, and Fig. 24 shows one pattern of sub-

\begin{tabular}{|l|l|l|l|l|l|l|l|}
\hline 11 & 2 & 5 & 6 & 3 & 4 & 7 & 8 \\
\hline 4 & 3 & 8 & 7 & 2 & 1 & 6 & 5 \\
\hline 2 & 1 & 6 & 5 & 4 & 3 & 8 & 7 \\
\hline 3 & 4 & 7 & 8 & 1 & 2 & 5 & 6 \\
\hline 5 & 6 & 1 & 2 & 7 & 8 & 3 & 4 \\
\hline 8 & 7 & 4 & 3 & 6 & 5 & 2 & 1 \\
\hline 6 & 5 & 2 & 1 & 8 & 7 & 4 & 3 \\
\hline 7 & 8 & 3 & 4 & 5 & 6 & 1 & 2 \\
\hline
\end{tabular}

Fig. 24.

\begin{tabular}{|c|c|c|c|c|c|c|c|}
\hline 17 & 257 & 619 & 1063 & ss & $1 / 83$ & we & \\
\hline & & 2098 & 2213 & 677 & 0.57 & 9 & 40,5 \\
\hline & 3 & $1+\infty$ & 455 & 34 & 9 & פ958 & 207 \\
\hline & & 23 & 2539 & 23 & 58 & $6 / 3$ & 160 \\
\hline & 859 & 233 & 41 & 2007 & 229 & | & 775 \\
\hline & & 557 & 170 & 1699 & loss & 881 & 650 \\
\hline & & $\mid 287$ & 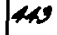 & $\$ 20$ & $\infty$ & 563 & דתו \\
\hline & & 203 & 285 & 9 & 853 & 227 & $\$ 7$ \\
\hline
\end{tabular}

Fig. 25.

See "Pandiagonal Prime Number Magic Squares," by Mr. Chas. D. Shuldham, The Monist, Oct. 1914, Vol. XXIV, No. 4. 
sidiary square, other patterns being easily found. The magic square shown in Fig. 25 was constructed from the above series with the subsidiary squares arranged according to Fig. 24 , one being revolved 90 degrees from the other.

THE SQUARE OF THE 9th ORDER.

It will be noted in Fig. 24 that each line, column and the two diagonals contain no like numbers. The numbers are also arranged

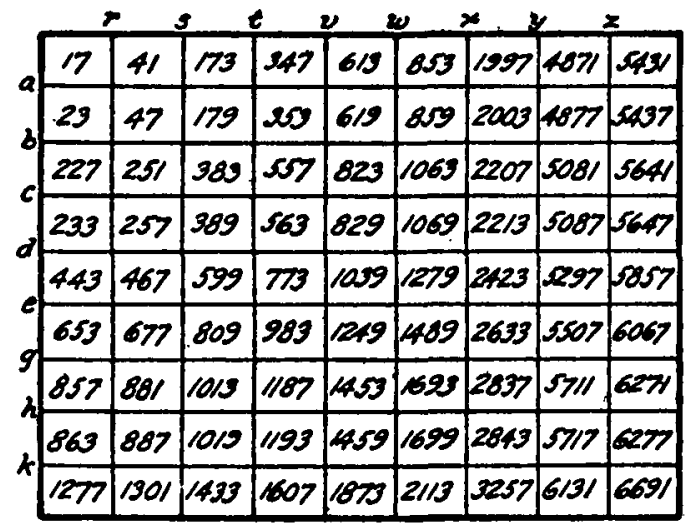

Fig. 26.

so that the pattern will set upon itself, by reflection or revolving, and not produce duplicate numbers in the final square. In like manner, the subsidiary squares for the 9th order square are ar-

\begin{tabular}{|l|l|l|l|l|l|l|l|l|}
\hline 9 & 8 & 4 & 7 & 6 & 2 & 5 & 1 & 9 \\
\hline 7 & 6 & 2 & 5 & 1 & 9 & 3 & 8 & 4 \\
\hline 5 & 1 & 9 & 3 & 8 & 4 & 7 & 6 & 2 \\
\hline 4 & 3 & 8 & 2 & 7 & 6 & 9 & 5 & 1 \\
\hline 2 & 7 & 6 & 9 & 5 & 1 & 4 & 3 & 8 \\
\hline 9 & 5 & 1 & 4 & 3 & 8 & 2 & 7 & 6 \\
\hline 8 & 4 & 3 & 6 & 2 & 7 & 1 & 9 & 5 \\
\hline 6 & 2 & 7 & 1 & 9 & 5 & 8 & 4 & 3 \\
\hline 1 & 9 & 5 & 8 & 4 & 3 & 6 & 2 & 7 \\
\hline
\end{tabular}

Fig. 27.

\begin{tabular}{|c|c|c|c|c|c|c|c|c|}
\hline $56 \times 1$ & 265 & 829 & 1187 & 2007 & 178 & 167 & 200 & e2s \\
\hline 201 & $4 \infty$ & 2000 & fort & 7 & 10079 & 557 & 537 & |205| \\
\hline 773 & 1871 & 100 & 251 & 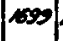 & 200 & $\alpha \pi$ & 105 & $6 / 9$ \\
\hline 230 & $\infty 23$ & 627 & 607 & |ars & 900 & 2113 & $a x=$ & 4 \\
\hline 859 & 2007 & 677 & 1277 & 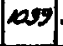 & $\sec \theta$ & 5007 & 300 & $1>8$ \\
\hline 603 & | & $=47$ & 069 & 200 & 807 & 23 & 400 & sasy \\
\hline $\cos$ & $\mathrm{sen}$ & 227 & 809 & 200 & SוI & 1998 & sor & 1270 \\
\hline 3ata & $\$ 7$ & 693 & $|6 / 3|$ & $|\operatorname{ses}|$ & $14+3$ & as & |sis & sear \\
\hline 78 & 100 & & $2+1$ & 257 & 1060 & פas & $s<0$ & 857 \\
\hline
\end{tabular}

Fig. 28.

ranged. Fig. 26 shows an irregular series, and Fig. 27 shows a pattern for the subsidiary squares, one being a reflection of the other. A resulting square is shown in Fig. 28. 
It may also be noted that, from Fig. 26, sets of numbers may be chosen for squares of the 4th, 5th, 7th, and 8th orders, and the possible number of distinct squares which may be formed from these depends on the laws of permutations and combinations.

TWIN SQUARES.

Two magic squares having like summations and having no numbers in common have been termed "twin squares."

\begin{tabular}{|l|l|l|l|}
\hline 1 & 599 & 701 & 207 \\
\hline 281 & 757 & 421 & 179 \\
\hline 547 & 71 & 209 & 631 \\
\hline 809 & 211 & 127 & 491 \\
\hline
\end{tabular}

\begin{tabular}{|c|c|c|c|}
\hline 29 & 619 & 677 & 313 \\
\hline 257 & 233 & 449 & 199 \\
\hline 529 & 47 & 409 & 659 \\
\hline 829 & 239 & 103 & 467 \\
\hline
\end{tabular}

Fig. 29.

To construct twin squares of the 4th order, we select a table of numbers having 8 or more columns and 4 numbers per column. The tabular differences may be irregular. From the top line of numbers, select two groups of four numbers each, that have like summations. Each of these groups will indicate the columns of numbers to be used in the respective squares. Each square is constructed as was explained in reference to Figs. 4, 5 and 6.

Twin squares of the 4th and 5th orders are shown in Figs. 29 and 30 respectively. In both examples, the above method was employed.

The foregoing methods of constructing prime number squares, by the use of tabular series, are obviously of little use for con-

\begin{tabular}{|l|l|l|l|l|}
\hline 389 & 643 & 6113 & 5327 & 1321 \\
\hline 5717 & 2161 & 179 & 133 & 5903 \\
\hline 223 & 5693 & 5507 & 1951 & 1019 \\
\hline 774 & 809 & 1060 & 5403 & 5297 \\
\hline 623 & 5087 & 1531 & 599 & 053 \\
\hline
\end{tabular}

\begin{tabular}{|l|l|l|l|l|}
\hline 2909 & 3501 & 653 & 1097 & 6151 \\
\hline 887 & 6991 & 2699 & 3373 & 463 \\
\hline 3169 & 233 & 677 & 6701 & 3559 \\
\hline 657 & 3329 & 1009 & 23 & 467 \\
\hline 863 & 257 & 6561 & 3119 & 5703 \\
\hline
\end{tabular}

Fig. 30.

structing squares of the higher orders, due to the increased diffculty in finding series of the necessary requirements. Large squares have been constructed, however, by other methods."

- See squares by Messrs. Shuldham and Muncey in The Monist, Oct. 1913, Vol. XXIII, No. 4, pp. 623 to 630. 


\section{PRIME NUMBER TABLES.}

To facilitate the finding of sets of prime numbers to be used in the construction of prime number magic squares, the prime number series has been arranged in tabular form in various ways.

One form of table is shown in Fig. 31, which is composed of a lattice having five cells in each line, the columnar length of the table depending on the range of numbers to be tabulated. The cells are counted by odd numbers in natural order from left to right, first through the top line, then through the second, and so on down through the table, a dot being placed in each cell where a prime number falls. In some cases, the table is made more convenient by extending it to form extra columns containing numbers which are duplicates of those in the table proper. Fig. 31 shows two supplementary columns which contain duplicates of the numbers in the first two columns. It should also be noted that the numbers in the supplementary columns must be one cell nearer the head of the table than the numbers they duplicate.

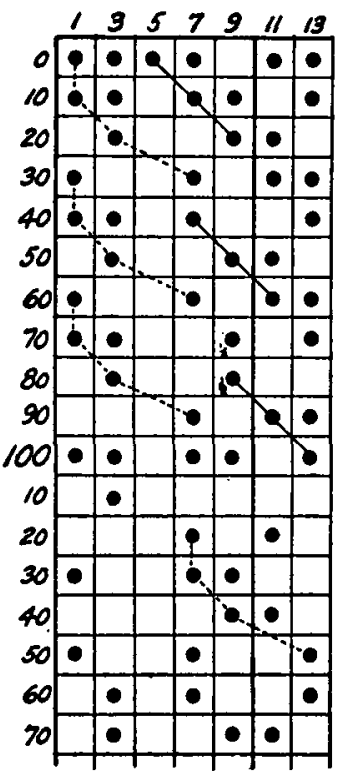

Fig. 31.

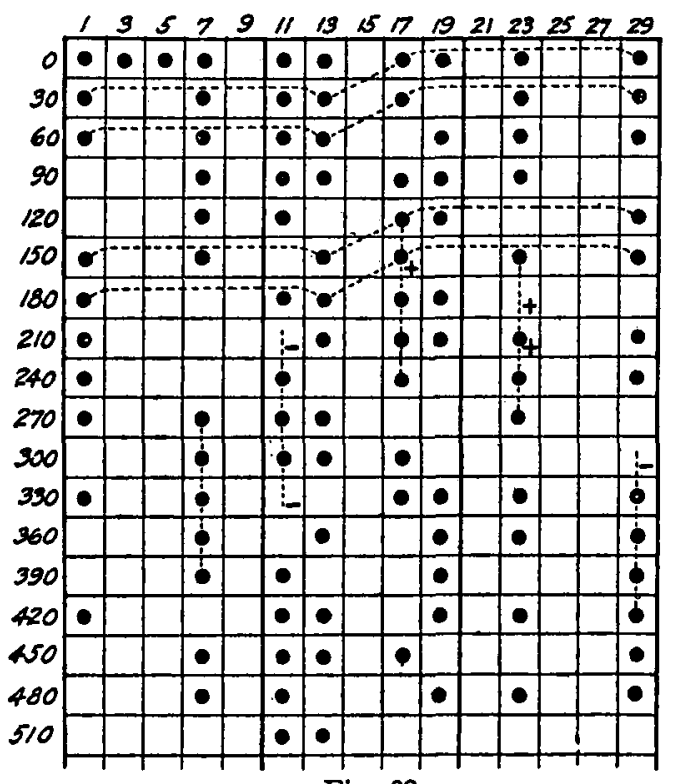

Fig. 32.

At the top of the table, the columns are numbered $1,3,5, \ldots$, and at the side, the lines are numbered $0,10,20,30, \ldots$, the latter numeration depending on the number of cells in a line. The value of a number represented by any dot, may be determined by adding 
together the index numbers at the end of the line and column in which the dot lies.

Fig. 32 shows another table in which the increments between lines are 30 ; and a portion of a still larger form of table is shown in Fig. 33, where the increments between lines are 210 .

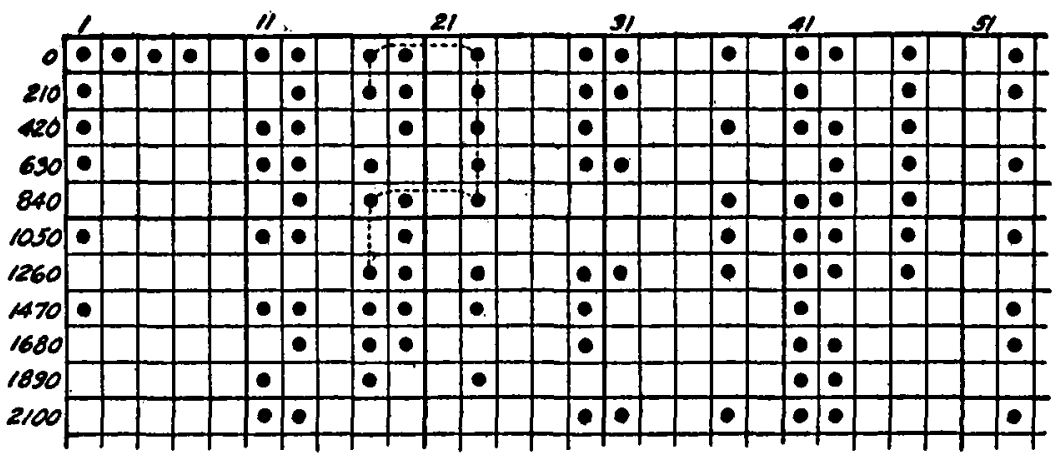

Fig. 33.

To get the best formation of tables, the increments between lines should be a multiple of two or more different small prime factors. In Fig. 31, the increment is $10=2 \times 5$; in Fig. 32, the increment is $30=2 \times 3 \times 5$; and in Fig. 33, the increment is $210=$ $2 \times 3 \times 5 \times 7$. The value of this may be observed by the resulting linear grouping of numbers in the tables.

To illustrate the use of these tables, we will point out a few of the groups of numbers which have been used in the preceding magic squares.

In Fig. 31 will be found three triads, the dots in each triad being indicated by a straight line connecting them. This group of 9 numbers is shown in Fig. 1 and is arranged in magic square formation in Fig. 3. It will be noted that the 9 dots in the table form a symmetrical figure, which indicates an associated group of numbers. In these forms of tables, all associated groups of numbers will show symmetrical figures, unless the grouping runs over the edge of the table. If the table in Fig. 31 had not been extended, the 9-number group would have appeared irregular if kept within the table.

In the same table will be found four irregular groups having four dots each, as indicated by dotted lines. Each group has the same form, though their relation is unsymmetrical. This group of 16 numbers was used to form the magic square shown in Fig. 6. 
A symmetrical group of 16 numbers is pointed out in Fig. 32, which was used to form the associated square in Fig. 8 and the pandiagonal square in Figs. 9 and 11.

In Fig. 33 is shown an irregular group of 9 numbers. Nine groups of this form were used in constructing the square shown in Fig. 28.

The foregoing rules of construction will aid in the simple formation of magic squares with prime numbers, but these rules are apparently inadequate in certain instances, for example, to construct a pandiagonal 9th order square, or to form a magic cube with prime numbers. The writer believes that these and other problems can be mastered if we bring further irregularities into the magic square series. In the following pages will be shown some of these irregularities, with an introduction of the "kink."

THE KINK.

The "kink" was first discovered in analyzing the prime number square, $S=102$. Fig. 34 shows this square resolved into two $\mathrm{La}$

\begin{tabular}{|c|c|c|c|}
\hline 3 & 71 & 5 & 23 \\
\hline 59 & 11 & 37 & 1 \\
\hline 77 & 13 & 41 & 31 \\
\hline 29 & 7 & 19 & 47 \\
\hline
\end{tabular}

$\mathrm{S}=102$

\begin{tabular}{|c|c|c|c|}
\hline 2 & 36 & 12 & 18 \\
\hline 12 & 18 & 2 & 36 \\
\hline 18 & 12 & 36 & 2 \\
\hline 36 & 2 & 18 & 12 \\
\hline
\end{tabular}

La Hireian Subsidiaries.

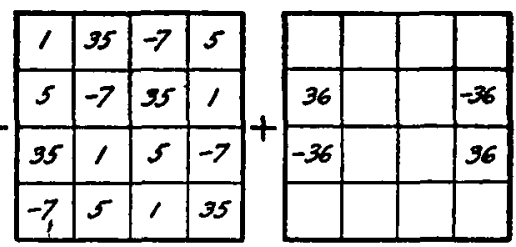

Kink.

Fig. 34.

Hireian subsidiaries of Jaina formation and a kink. It will be noticed that this kink involves four cells, rectangular in position, and of equal numeral values, two being plus and two minus. For

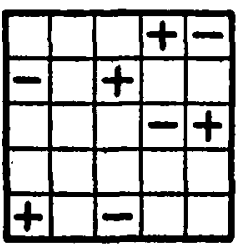

A

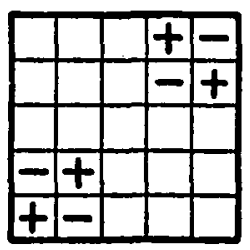

B

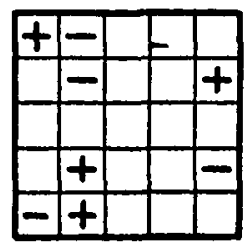

C

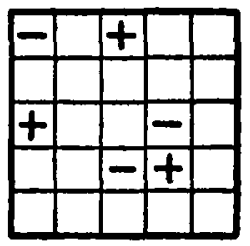

D

Fig. 35.

convenience of designation we will give this form the term rectangular kink. It will be observed in the rectangular kink, that any two values in a horizontal or vertical line, counteract each other; there- 
fore, a rectangular kink of any plus or minus values, may be added to any magic square without affecting the magic summations, providing any part of the kink does not fall in a magic diagonal. A rectangular kink may affect a diagonal, providing a second kink is added to correct the fault. Fig. 35 illustrates a few double rectangular kinks by which the main diagonals of the square have been corrected. In the central cell of the square " $D$," two kink values are neutralized, and are therefore not shown.

Two like rectangular kinks may be combined so as to form a kink that will not destroy the values of any of the magic summations of a pandiagonal square. This form might be termed pan-

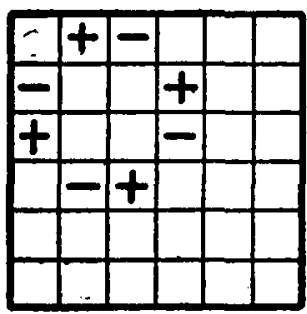

A

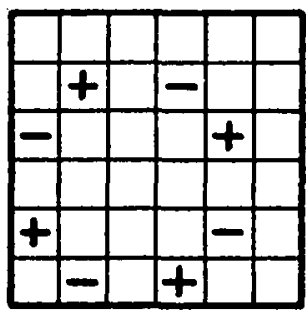

B

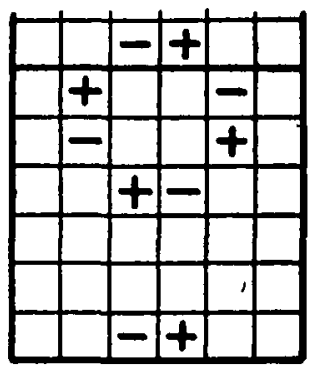

C

Fig. 36.

diagonal or octagonal kink. Fig. 36 shows a few of the latter type, the square " $C$ " showing how the octagonal form may be apparently missing in some cases, due to the kink running over the edge of the square.

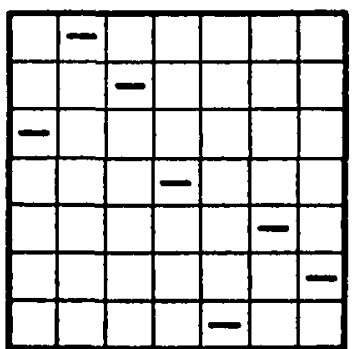

A

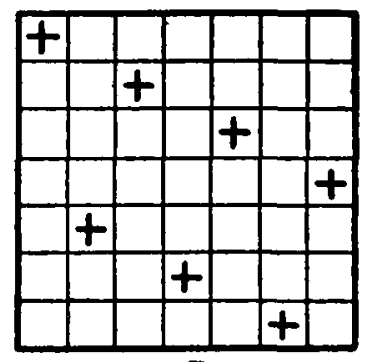

B

Fig. 37.

minus or plus as shown in Fig. 37. In square " $A$ " the same value is taken from each line, column, and the two diagonals, which

There are also other kinks where all the values are either would mean a lowering of the summations but would not destroy 
its principal magic qualities. In square " $B$ " each line, column and all diagonals are affected alike, and may therefore be classed as a pandiagonal kink. The values in this latter kink are shown to run in a knight's path, which formation has suggested the term path kink.

When applying kinks to squares, it is not necessary that the same numeral values are applied to all the kinks involved. This

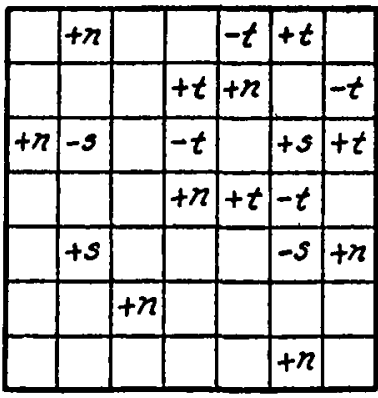

Fig. 38.

\begin{tabular}{|c|c|c|c|c|}
\hline 10 & 18 & 1 & 14 & 22 \\
\hline 11 & 24 & 7 & 20 & 3 \\
\hline 17 & 5 & 13 & 21 & 9 \\
\hline 23 & 6 & 19 & 2 & 15 \\
\hline 4 & 12 & 25 & 8 & 16 \\
\hline
\end{tabular}

Fig. 39.

\begin{tabular}{|c|c|c|c|c|}
\hline 1 & 2 & 3 & 4 & 5 \\
\hline 6 & 7 & 8 & 9 & 10 \\
\hline 11 & 12 & 13 & 14 & 15 \\
\hline 16 & 7 & 18 & 19 & 20 \\
\hline 21 & 22 & 23 & 24 & 25 \\
\hline
\end{tabular}

Fig. 40.

is illustrated in Fig. 38, where are shown a rectangular, an octagonal, and a path kink. It can be realized from this illustration that a magic square series can be greatly distorted by the addition of a few kinks.

The following example will suffice to show the constructive application of the kinks. Fig. 39 is a magic square containing the

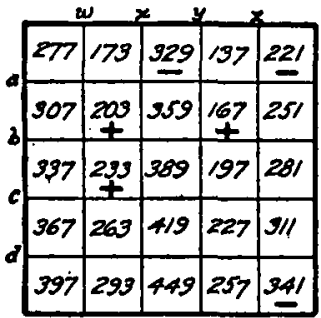

Fig. 41.

\begin{tabular}{|c|c|c|c|c|}
\hline 277 & 173 & 179 & 137 & 71 \\
\hline 307 & 353 & 359 & 317 & 251 \\
\hline 397 & 389 & 389 & 197 & 281 \\
\hline 367 & 263 & 419 & 227 & 311 \\
\hline 397 & 293 & 449 & 257 & 191 \\
\hline
\end{tabular}

Fig. 42.

\begin{tabular}{|l|l|l|l|l|}
\hline 251 & 419 & 277 & 197 & 293 \\
\hline 307 & 257 & 353 & 311 & 179 \\
\hline 263 & 71 & 389 & 397 & 317 \\
\hline 449 & 307 & 227 & 173 & 281 \\
\hline 137 & 363 & 191 & 359 & 367 \\
\hline
\end{tabular}

Fig. 43.

series $1,2,3, \ldots n^{2}$, constructed with subsidiaries of the pattern shown in Fig. 13. The numbers of this square are shown in tabular form in Fig. 40. The position of two arbitrary rectangular kinks has been designated in Fig. 39, and these kinks have been transferred to the same respective numbers in Fig. 40. Any set of numbers with tabular differences of $a, b, c, d$, and $w, x, y, z$, that is affected by kinks, as shown in Fig. 40, is susceptible to transformation into a 
magic square. In Fig. 32 are shown by vertical dotted lines, five sets of numbers which have been tabulated in Fig. 41, the strings of the former being arranged as columns in the latter. The selections in Fig. 32 were made with the kinks in mind, which are indicated with small plus or minus signs. Where the minus signs occur, we have substituted the number five cells above in the same column, and where the plus signs occur, the number five cells below has been used; these differences of position being equal to 150 , which is the numeral value of the kink used. This kink value has been added to the cells indicated in Fig. 41 which gives the numbers shown in Fig. 42. The numbers in Fig. 42 are now transposed into the magic square Fig. 43 , in the same respective order that the numbers of Fig. 40 are shown in Fig. 39.

It can be seen from the foregoing that unusual irregular series, formed by the use of kinks, may be anticipated, and in a great many cases, found and arranged in magic squares. The writer believes that the $3 \mathrm{~d}$ order cube will be solved in this manner, which will be referred to in the following pages.

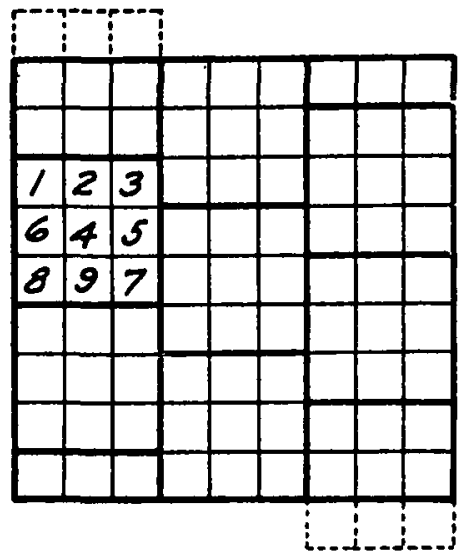

Fig. 44.

\begin{tabular}{|c|c|c|c|c|c|c|c|c|}
\hline 7 & $|1069|$ & 601 & 261 & 269 & 401 & 11 & 577 & 108 \\
\hline & 313 & & & & & 9 & 81 & \\
\hline & 101 & 5 & & & 39 & 7 & 281 & \\
\hline & $(351)$ & 371) & ?3) & 99 & 1 & 17 & 73 & \\
\hline & 811 & 569 & . & & 7 & 509 & 69 & $(137$ \\
\hline & 5 & 3 & . & 77 & 51 & $(1547)$ & 83 & \\
\hline & 73 & 83. & $1 / 5\rangle$ & 9 & 459 & $A+3$ & 293 & 0 \\
\hline & 1 & 557 & 1 & 11 & 597 & gest & 977 & 10 \\
\hline & & st & & 19 & 33 & 607 & 49 & \\
\hline
\end{tabular}

Fig. 45.

Another method of using the kink, is to construct the desired magic using as few composite numbers as possible, and then add various kinks at the places where the composite numbers occur; the process being continued until all the composite numbers are eliminated. The pandiagonal square of the 9th order will undoubtedly be accomplished in this way.

For this square, the preliminary series (containing as few 
composites as convenient) should be so chosen as to allow each of the two subsidiary groups of numbers to be divided into three triads of equal values. The numbers are arranged in quarrels in the subsidiary squares, as shown in the pattern, Fig. 44, the triads being placed in vertical strings. The subsidiary squares are similar in pattern and are placed 90 degrees apart, or, one subsidiary may be reflected on either of its two diagonals.

Fig. 45 illustrates a pandiagonal square constructed by Chas. D. Shuldham. By the above method he has succeeded in transforming it to contain as low as six composites, which are indicated by circles.

\section{THE CUBE.}

To the writer's knowledge, the prime number cube of the $3 \mathrm{~d}$ order has not yet been constructed. The kink will undoubtedly aid in its construction, and the following example will suffice to show the application of the kink to the cube.

Fig. 46 shows the three respective layers of one of the various magic cubes constructed with the natural series of numbers, and

\begin{tabular}{|c|c|c|}
\hline 4 & 26 & 12 \\
\hline 17 & 3 & 22 \\
\hline 71 & 13 & 8 \\
\hline 18 & 1 & 23 \\
\hline 19 & 14 & 9 \\
\hline 5 & 27 & 10 \\
\hline 20 & 15 & 7 \\
\hline 6 & 25 & 11 \\
\hline 16 & 2 & 24 \\
\hline
\end{tabular}

Fig. 46.

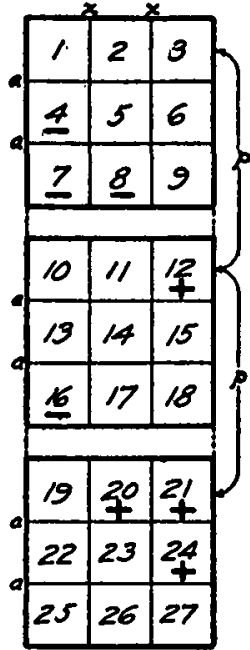

Fig. 47.

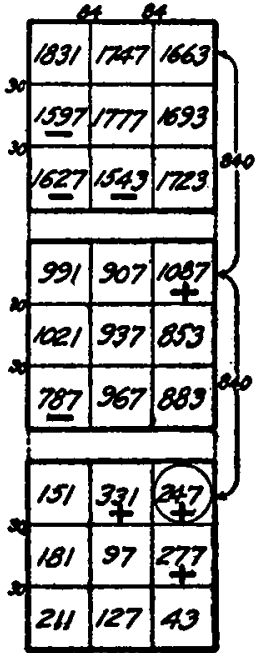

Fig. 48.

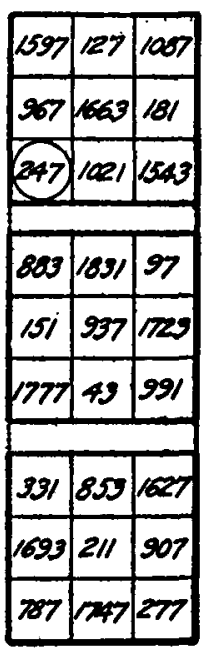

Fig. 49.

Fig. 47 shows this series in tabular form. A magic cube of ordinary construction must have a series whose tabular differences are $a, a$ and $p, p$ and $x, x$, as indicated in Fig. 47, the numbers being arranged in the cube in the same order as any cube of a straight series. 
The simple cubic kink is shown diagrammatically in Fig. 50 and has the form of a right parallelopiped. A single kink of this form can be added to the $3 \mathrm{~d}$ order cube in only one way, that is, its eight values must fall in the eight corner cells of the cube, otherwise the summation of the diagonals would be altered.

The position of a simple kink is indicated in Fig. 46, and is transposed to the same respective numbers in Fig. 47.

The series of prime numbers for the cube would be discovered in the same manner as were the numbers in Figs. 42 and 43 , that is, find any set of numbers in the prime number table that has tabular differences, as indicated by letters in Fig. 47, disregarding any composite numbers that may occur where the kink is indicated. For the plus values of the kink, a new set of values is discovered in the table which has the same geometric relation to each other as the originals. This is diagrammatically illustrated in Fig. 51. Care should be taken that a new set for the minus values can also be found in a symmetrically opposite direction.

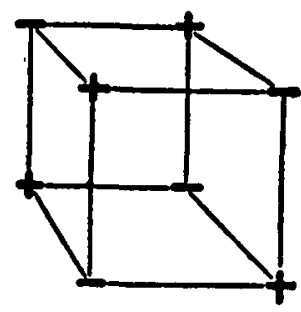

Fig. 50.
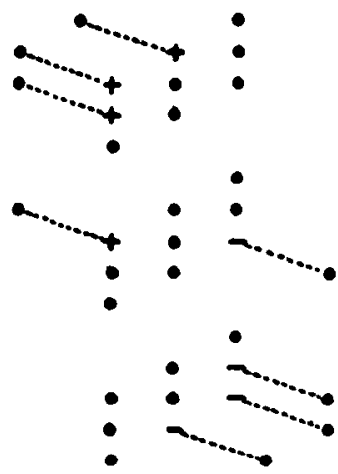

Fig. 51.

According to the above method, the writer has succeeded in finding, in a table of the form shown in Fig. 32, the series shown in Fig. 48, which contains the one composite number indicated with a circle. These numbers being transposed into a cube according to Figs. 46 and 47, produces the magic cube shown in Fig. 49.

Combinations of kinks can be added to cubes in various ways which the reader can easily discover for himself. The variations in tabular differences and in kink formation and combinations would apparently indicate that the discovery of a prime number series for a cube is possible. There is greater freedom in the 
application of kinks to the 4th order cube, though the writer has not investigated beyond the $3 \mathrm{~d}$ order.

It also seems possible that the cube of the 4th order may be constructed by an extension of the method of pseudo-complementaries. $^{\mathrm{s}}$

Patience and perseverance will be found to be the principal requirements in solving these difficult problems in prime number magics. Who will claim the honor of being the first $t n$ solve them?

Harry A. Sayles.

Schenectady, N. Y.

\section{BOOK REVIEWS.}

The Elementary Forms of the Religious Life. A study in religious sociology. By Emile Durkheim, professor of the faculty of letters at the University of Paris. Translated by J.W. Swain, M.A. London, George Allen \& Unwin, 1916. Price 15s. net.

This book must be valued from three different points of view. It contains reinterpretations of the principal social phenomena of primitive peoples; it contains a theory of the genesis of knowledge with doubtful philosophic implications; and it contains what we may assume for the present to be $M$. Durkheim's definitive pronouncement on the nature and the future of religion. All of these strands of argument are bound together by M. Durkheim's wellknown theory of the group-consciousness, but this theory itself must be assigned different values in these three developments.

It is in the more purely anthropological aspect that this book is most successful. Here M. Durkheim's views must be judged in comparison with those of the older interpreters such as Tylor, Müller, Lang, Frazer, Jevons, Robertson Smith, Mannhardt. As in most works of the sort, the author is most convincing when he sticks closest to the facts, when he is least metaphysical, and when he is engaged in refuting his predecessors. In fact, he is most convincing when he is showing us what the phenomena of primitive religion do not mean. M. Durkheim confines his observation almost entirely to Australia, and his theory of Australian totemism is distinctly the best that has yet been evolved. Why? Because he is able to show that totemism is not animal worship, that it is not derivative from ancestor worship, or from the "nature cult"; the totem is not a name; the group totem is not, as Frazer holds, a development from the conception totem. M. Durkheim's theory is the best because it is the nearest to being no theory at all. And when he comes

- See "Even Order Magic Squares with Prime Numbers," The Monist, Jan. 1916, Vol. XXVI, No. 1 . 